\title{
Design and Modelling of Graphene Field-Effect Transistor for Ultrasensitive Detection in Biosensor Application
}

\author{
Anis Amirah Alim, Reena Sri Selvarajan, Azrul Azlan Hamzah* \\ Institute of Microengineering and Nanoelectronics (IMEN), \\ Universiti Kebangsaan Malaysia (UKM) \\ Selangor, Malaysia \\ *azlanhamzah@ukm.edu.my
}

\begin{abstract}
The coronavirus disease is a pandemic attack and gravely weakening the ability of the global health community. Early rapid detection of a device has become the golden strategy to contain the virus due to no specific drugs or vaccines. Conventional methods to detect this disease are still involved with laborious procedures, time-consuming as well as expensive. In this paper, graphene field-effect transistor (GFET) is shown as a great potential for rapid and high sensitivity biosensor. However, the performance of the GFET is determined by various factors. Computational method is performed to minimize the complexity of the fabrication. The present work shows the simulation of embedded graphene based on length, metal contacts and Fermi Level which effects the electrical characteristic of GFET via Lumerical DEVICE charge solver simulation. The graphene conducting channel length and Fermi Level shows an abundance of effects on the ambipolar curve of GFET. Interface between graphene and metal contacts affect the transport of charge carriers in the device. Therefore, to determine optimal GFET efficiency and at the same time, minimize manufacturing costs, the requirement for system simulation is crucial.
\end{abstract}

Keywords-GFET, ultrasensitive detection, biosensor

\section{INTRODUCTION}

The race towards the development of user-friendly, portable, fast-detection and low-cost devices for healthcare system has become the primary demand for practical screening actions globally since the pandemic attack in December 2019 which known as Coronavirus disease [1]. Coronavirus disease 2019 (COVID-19) is a new infectious disease which associates with severe respiratory distress. Furthermore, with the absence of specific drugs or vaccines, early detection and management are crucial for containing the outbreak [2]. However, conventional methods using lateral flow assay (LFA) [3], loopmediated isothermal amplification (LAMP) [4], enzyme-linked immunosorbent assay (ELISA) [5] and reverse transcript polymerase chain reaction (RT-PCR) [6] involve with tedious and time-consuming method [7]. In this situation, a rapid and straightforward, one-step device that provides application at points of care testing (POCT) plays an important role. With the implementation of the biosensor as POCT, this will enable a faster strategy to prevent the spread. This biosensor acts as an analytical tool to monitor dynamics and interactions of biological activities such as attachment of biomolecules or pathogens and transfer the results into electrical signals [8]. Figure 1 explains the essential components in a biosensor.

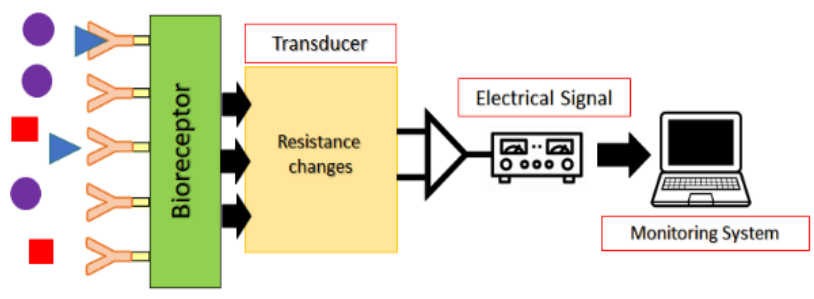

Fig. 1. Components in a biosensor [9].

With the emerging of micro/nanotechnologies, field-effect transistor (FET) could also be applied as a biosensor. This device has received widespread attention due to the attractive features such as ultra-sensitive detection, mass production capability, fast-result and low-cost manufacturing [10]. Seo et al. able to detect the presence of the virus spike protein by using graphene FET (GFET) in the clinical sample without any tedious clinical procedures [2]. Graphene is chosen for the sensing material due to its high electron mobility [11], transparency [12] and mechanical strength [13]. Various studies have been reported shown FET-based on graphene have potential as higher sensitivity and selectivity sensor [14-16]. Based on Campos et al. [17], the GFET can detect biomolecule down to attomolar concentration. With this advantage, the researcher able to achieve instantaneous measurement using small amounts of analytes.

In this paper, we introduce Lumerical Device CHARGE Solver for modelling and design of the GFET. Although the simulation only uses a semi-classical solver to calculate the drift-diffusion in complex geometries, the model results still produce reasonable accuracy and can be used as a good first 
approximation. To observe the performance of the GFET for biosensor application, several parameters are being taken account. This process is vital to minimise the cost of fabrication and at the same time, speed up the manufacturing process.

\section{THEORETICAL EXPLANATION}

The characteristics and principle applied for the graphene field-effect transistor (GFET) are explained in this part. In this study, we use Landauer Buttiker formula to understand the effect of specific parameter towards the conductivity of GFET.

\section{A. Principle of Graphene Field-Effect Transistor}

The FET biosensors operation are derived from the basic working principle of metal-oxide-semiconductor FET (MOSFET). The fundamental active component for charge carriers to flow in MOSFET is the semiconductor substrate (silicon). On top of the silicon, a thin insulation (dielectric) sheet which covered by three metallic contacts: drain (D), source $(\mathrm{S})$ and gate $(\mathrm{G})$ electrodes. MOSFET operates when the charge carriers consist of electron and holes form a channel (depletion region) from the source to the drain. The formation of the depletion region is controlled by the gate voltage located in the middle of the MOSFET [18]. The same principles are applied to the FET-based biosensor by changing the gate potential value. The gate surface of the FET-based biosensors is functionalised with a biological recognition feature capturing the target molecule that shifts the electrostatic gating effect. A recent review has shown that graphene has high potential as the sensing material for gate channel [19]. See figure 2 below.

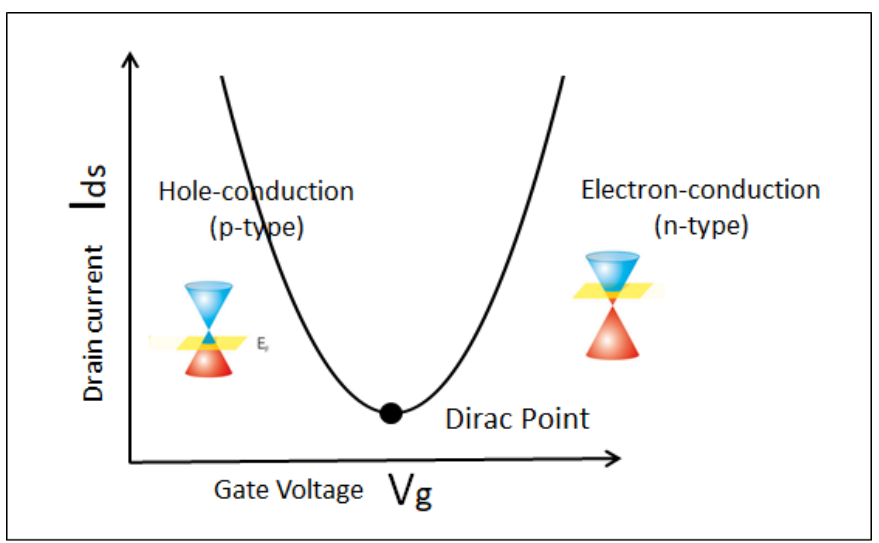

Fig. 2. Transfer characteristic of graphene.

Graphene presents a peculiar behaviour where energy bands form two circular cones with negative (valence) and positive (conduction) energy bands touch at a point called Dirac point (k-point). Due to this condition, all graphene electrons have the same velocity and absolutely no inertia and act as massless Dirac particles [20]. This property induces ambipolar curve behaviour (electron and hole). The density of the charge carrier in the graphene can be modified by the fieldeffect using the backgate and consequently the conductivity. When no gate voltage applied, the Dirac point is located at the
Fermi level. Tuning the Fermi level above Dirac point, causing the conduction band loaded with electrons (n-type conduction) and inversely adjusting, causing the valence band to filled with holes (p-type conduction)[21]. We can observe the conductivity of the graphene channel driven by charge carries at the ambipolar curve, as shown in Figure 2. The point of lowest current is known as the Dirac point. The value of the Dirac point is affected by various parameters such as contact resistance [22], doping on the graphene [23] and channel length [24]. Therefore, the performance of the GFET is observed based on the current output $\left(\mathrm{I}_{\mathrm{ds}}\right)$.

\section{B. Landauer Buttiker Formula and Its Application}

The conductivity of FET is controlled by the gate voltage, which induces electrical field in the channel, thus alter the carrier density. Computational approximation for this mathematical application is performed to understand the performance of the GFET based on the Landauer Buttiker formula [23],

$$
I(A)=\frac{2 q}{\hbar} \int_{-\infty}^{\infty} T(E) M(E)\left(f_{1}-f_{2}\right) \cdot d E
$$

Here, $\mathrm{h}$ is the Planck's constant, $q$ is the charge, $\mathrm{f}_{1}$ is the fermi energy level for undoped graphene, and $f_{2}$ is the fermi energy level after doping. The $\mathrm{T}(\mathrm{E})$ is the transport of charge carrier between two electrodes given by,

$$
T(E)=\frac{M(E)}{M(E)+L}
$$

Where $\lambda(E)$ is the mean free path and $\mathrm{L}$ is the channel length. From equation (2), the channel length is inversely proportional to current output. The $\mathrm{M}(\mathrm{E})$ is the number of modes given by,

$$
M(E)=\frac{2 W E}{\pi h v_{F}}
$$

Whereas $\mathrm{W}=$ width of the channel, $\mathrm{E}$ is the energy level, $\mathrm{vF}=106 \mathrm{~m} / \mathrm{s}$ [25]. Doping induces significant changes to the conductivity of the GFET. By doping, graphene Fermi level is shifted, thus change its electronic properties. "Doped" any materials onto graphene, means they added or subtracted electrons from graphene by chemical means. The charge transfers between the graphene and the dopant changes the graphene work function (WF), $\Phi$. The WF of any material can be described as the energy needed to detach the electron from the maximum filling stage in the Fermi solid-to-vacuum distribution (i.e. stationary in a field-free region just outside the solid) at absolute zero. It is a basic electrical property of any substance which gives an understanding of the relative location of the surface of Fermi level. The formula for work function stated as, 


$$
W F(e V)=E_{\text {Vae }}-E_{f}
$$

Whereas WF is the work function, Evac is the energy level at vacuum and Ef is fermi energy level. It this condition, the WF of graphene is changed based on the fermi level shifting [26]. Thus, induces difference current output based on equation (1). Doping also increase the carrier density $\left(n_{g}\right)$ in the channel given by,

$$
n_{g}=\frac{1}{\pi}\left(\frac{E}{w_{p}}\right)^{2}
$$

Based on equation (1), we can be simplified that the current output is proportional to $n_{\varepsilon}$. CHARGE calculates the driftdiffusion based on the carrier density of the model in graphene whereas electron density (n) is equal to holes density (p) [25].

Electrical transfer in the GFET requires two metal electrodes at both ends. Thus, full understanding of physics for the metal-graphene interfaces is import. Giovannetti et.al [27] use first-principle calculations to analyse graphene adsorption in a sequence of metal substrates by using density functional theory (DFT). Based on the study, these 4 materials (Al, Cu, $\mathrm{Ag}$ and $\mathrm{Au}$ ) preserved weak bonding and allows the Fermi level to deviate significantly from the Dirac point of the graphene, leading in a doping mechanism [26]. The symbol for the sum of doping is concluded from the differential between the WF of the metal graphene. Because of the difference in WF between the graphene and metal surfaces, as soon the both material in contact of each other, electrons are transferred from one to the other to achieve equilibrium at the Fermi levels [25]. Thus, the difference in fermi level contributes the conductivity of the GFET obeys the equation (1).

\section{LUMERICAL SIMULATION}

The present works shows the model of graphene field-effect transistor (GFET) using LUMERICAL's Device CHARGE Transport Simulation. CHARGE conscience solves the system of equations defining the electrostatic potential (Poisson's equations) and the free carrier density (drift-diffusion equations)[28]. Graphene material was not available in the material library and has been modelled based on parameter in Sharma et. al [23]. The work function of undoped single layer graphene is set at 4.6 [26] with carrier mobility of $6000 \mathrm{~cm}^{2} / \mathrm{V}$ is set the same for both holes and electrons at thermal equilibrium of $300 \mathrm{~K}$ [29]. See figure 3 below.

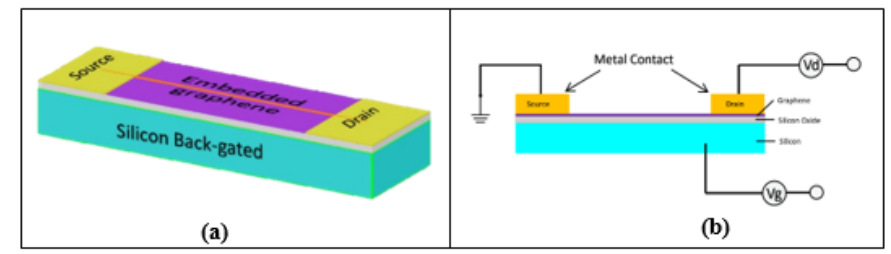

Fig. 3. Model of graphene field-effect transistor (a) Model in Lumerical Software (b) Schematic diagram.

The device is modelled in steady-state condition with symmetric metal contact. The thickness of metal contact (electrode) and silicon dioxide is fixed at $60 \mathrm{~nm}$ and $300 \mathrm{~nm}$, respectively. The single layer graphene is placed on top of the silicon dioxide and highly doped n-type silicon. Work function and band gap of graphene is set based on the doping mechanism [23]. The metal electrode work function is set based on the this work for $\mathrm{Au}, \mathrm{Cu}, \mathrm{Al}$ and $\mathrm{Ag}$ [27]. Table 1 shows the parameter range in the simulation to analyse the electrical characteristics of GFET.

TABLE I. PARAMETER RANGE IN SIMULATION.

\begin{tabular}{|l|l|}
\hline \multicolumn{1}{|c|}{ Parameter } & \multicolumn{1}{c|}{ Range } \\
\hline Channel Length $(\mu \mathrm{m})$ & $6-30$ \\
\hline Work Function of graphene $(\mathrm{eV})$ & $4.04-5.04$ \\
\hline Metal contact work function $(\mathrm{eV})$ & $4.04-4.74$ \\
\hline Electrode size $(\mu \mathrm{m})$ & $2.0 \times 3.0$ \\
\hline
\end{tabular}

\section{RESULTS AND ANALYSIS}

\section{A. Work function and Fermi Level of Doped Graphene}

Based on Figure 4, the graph is observed based on the location of Dirac point at the lowest current drain, Id. The transfer characteristics curve of GFET achieve ambipolar curve which proven the distinctive ability of graphene. See figure 4 below.

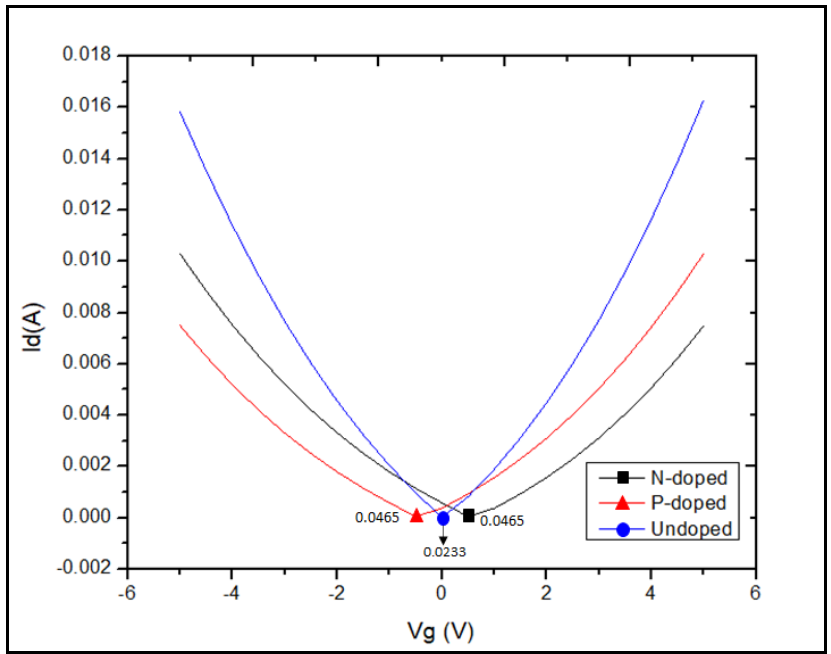

Fig. 4. Transfer characteristics curve of GFET for n-doped, p-doped and undoped graphene sheet. 
Doping affects the current output to increase slightly but causing the ambipolar curve to be asymmetry. Nitrogen doping (n-doped) cause the Fermi-level of graphene shift upwards due to electron-doping and decrease the work function of graphene [30]. On the contrary, boron doping (p-doped) causes the Fermi level of graphene to shift downward due to hole-doping. Therefore, the work function of graphene increase. The N-type and P-type of graphene can be observed from the electronic properties at the Dirac point. P-type graphene causing the ambipolar curve Dirac point shift to the left whereas N-type graphene causing the ambipolar curve Dirac point shift to the right [31]. This condition obeys the principle stated by Landauer Buttiker formula as in equation (1). Doping also increasing the band gap and the effective mass as explained in equation (5)

\section{B. Channel Length}

The variation of channel length is tested for the undoped graphene whereas the Dirac point is located at $\mathrm{Vg}=0$. Based on Figure 5, the symmetrical ambipolar curve performs distinction results from varied channel length. Based on the equation (2), channel length contributes major impact towards the conductivity of the GFET which influence by the transport charge carrier between the electrodes. The current output is inversely proportional to the channel length. Longer channel length also promotes higher resistance [32].

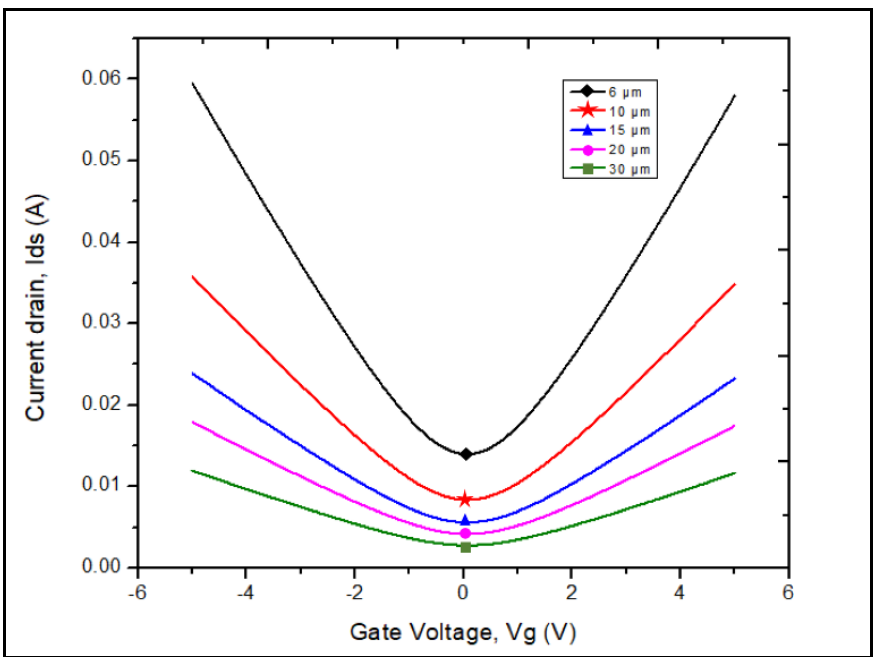

Fig. 5. Transfer characteristics of GFET for five different channel length.

\section{Material of the Contact}

Graphene FET requires metal contacts to allow the current flows in the graphene. The electrical properties of the GFET is observed on the average current output at the Dirac point. The material tested for this simulation is involves with $\mathrm{Au}, \mathrm{Cu}, \mathrm{Ag}$ and $\mathrm{Al}$ as metal contacts. The difference in work function causing the mismatch between fermi level at the metalgraphene interface thus affect the transport charge carrier between electrodes following the principle in equation (1). $\mathrm{Au}$ performs the best conductivity for metal electrodes as shown in
Figure 6 due to its higher work function compare to graphene thus ease the transfer of electron from graphene to the metal [27].

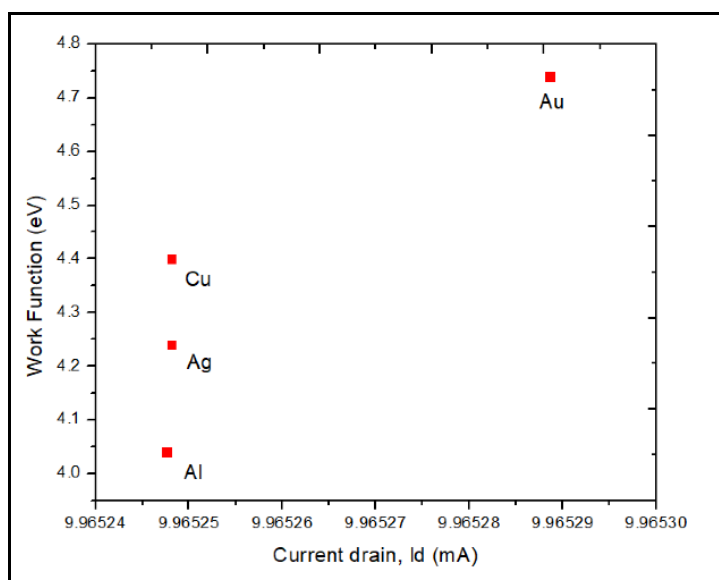

Fig. 6. Average current drain of Dirac points response as to the metal work function.

\section{CONCLUSION}

Lumerical Device CHARGE Solver simulation shows a great potential for semi-classical calculation of the graphene field-effect transistor. In this paper, we found that channel length, fermi-level and metal contacts play a crucial role in conductivity of the GFET which have be proven by using Landauer Buttiker formula. This formula provides fundamental understanding toward the physics of GFET. Using CHARGE also enable us to find a better design in order to minimize the complexity of fabrications. The model result although not physically precise, the charge carrier transport of GFET is still acceptable and provide a better approximation before fabrication. Thus, this process will able to reduce the cost of fabrication and at the same time, speed up the fabrication process.

\section{ACKNOWLEDGMENT}

This research is funded by AKU-254 HICoE MEMS grant funded by Ministry of Higher Education (MOHE) Malaysia.

\section{REFERENCES}

[1] I. Santiago, "Trends and Innovations in Biosensors for COVID-19 Mass Testing," ChemBioChem, 2020.

[2] G. Seo, G. Lee, M.J. Kim, S.H. Baek, M. Choi, and K.B. Ku, "Rapid Detection of COVID-19 Causative Virus (SARS-CoV-2) in Human Nasopharyngeal Swab Specimens Using Field-Effect Transistor-Based Biosensor," ACS Nano. 2020.

[3] H. Ma, W. Zeng, H. He, D. Zhao, Y. Yang, and D. Jiang, "COVID-19 diagnosis and study of serum SARS-CoV-2 specific IgA, IgM and IgG by a quantitative and sensitive immunoassay," medRxiv, 2020. 
[4] J. Kashir and A. Yaqinuddin, "Loop mediated isothermal amplification (LAMP) assays as a rapid diagnostic for COVID-19," Med Hypotheses, 2020.

[5] L. Guo, L. Ren, S. Yang, M. Xiao, D. Chang, and F. Yang, "Profiling Early Humoral Response to Diagnose Novel Coronavirus Disease (COVID-19)," Clin Infect Dis., 2020.

[6] J. Mo, M. Angelichio, L. Gow, V. Leathers, and M.W. Jackwood, "Validation of specific quantitative real-time RT-PCR assay panel for Infectious Bronchitis using synthetic DNA standards and clinical specimens," J Virol Methods, 276, 2020.

[7] A. Tahamtan and A. Ardebili, "Real-time RT-PCR in COVID-19 detection: issues affecting the results," Expert Review of Molecular Diagnostics, 2020.

[8] A.T. Lawal, C.I.L. Justino, A.R. Gomes, A.C. Freitas, A.C. Duarte, and T.A.P. Rocha-Santos, "Progress in utilisation of graphene for electrochemical biosensors,” TrAC - Trends Anal Chem, vol. 91, pp. 53-66, 2017.

[9] R. Vargas-Bernal, E. Rodrguez-Miranda, and G. Herrera-Prez "Evolution and Expectations of Enzymatic Biosensors for Pesticides," Pesticides - Advances in Chemical and Botanical Pesticides, 2012.

[10] P. Visconti, A. Della Torre, G. Maruccio, E. D’Amone, A. Bramanti, and R. Cingolani, "The fabrication of sub-10 nm planar electrodes and their use for a molecule-based transistor," Nanotechnology, 2004.

[11] W. Zhu, V. Perebeinos, M. Freitag, and P. Avouris, "Carrier scattering, mobilities, and electrostatic potential in monolayer, bilayer, and trilayer graphene," Phys Rev B - Condens Matter Mater Phys., 2009.

[12] Z. Li, W. Zhang, and F. Xing, "Graphene Optical Biosensors," International journal of molecular sciences, 2019.

[13] J.W. Jiang. "Graphene versus MoS2: A short review," Front Phys., vol. 10, no. 3, pp. 287-302, 2015.

[14] W. Fu, 1. Jiang, E.P. van Geest, L.M.C. Lima, and G.F. Schneider, "Sensing at the Surface of Graphene Field-Effect Transistors," Advanced Materials, vol. 29, 2017.

[15] C.I.L. Justino, A.R. Gomes, A.C. Freitas, A.C. Duarte, T.A.P. RochaSantos, "Graphene based sensors and biosensors," TrAC - Trends Anal Chem, vol. 91, pp. 53-66, 2017.

[16] A.T. Lawal, "Progress in utilisation of graphene for electrochemical biosensors," Biosens Bioelectron, vol. 106, pp. 149-78, 2018.

[17] R. Campos, J. Borme, J.R. Guerreiro, G. Machado, M.F. Cerqueira, and D.Y. Petrovykh, "Attomolar Label-Free Detection of DNA Hybridization with Electrolyte-Gated Graphene Field-Effect Transistors," ACS Sensors, vol. 4, pp. 286-93, 2019.
[18] T. Agarwal, "What is the MOSFET: Basics, Working Principle and Applications," El-Pro-Cus, 2017.

[19] A.A. Hamzah, R.S. Selvarajan, and B.Y. Majlis, "Graphene for biomedical applications: A review," Sains Malaysiana, vol. 46, no. 7, pp. 1125-39, 2017

[20] A.K. Geim, and K. Novoselove, "The rise of graphene," Nat Mater., vol. 6, pp. 183-91, 2007.

[21] N.M.R. Peres, "Graphene: New physics in two dimensions," Europhys News, vol. 40, no. 3, pp. 17-20, 2009.

[22] F. Giubileo and A. Di Bartolomeo, "The role of contact resistance in graphene field-effect devices," Prog Surf Sci, vol. 92, no. 3, pp. 143-75, 2017.

[23] P. Sharma, S. Singh, S. Gupta, and I. Kaur, "Enhancing linearity in I-V characteristics by $\mathrm{B} / \mathrm{N}$ doping in graphene for communication devices," J Mater Sci Mater Electron, vol. 28, no. 11, pp. 7668-76, 2017.

[24] S.J. Han, Z. Chen, A.A. Bol, and Y. Sun, "Channel-length-dependent transport behaviors of graphene field-effect transistors," IEEE Electron Device Lett., vol. 32, no. 6, pp. 812-4, 2011.

[25] F. Giubileo, and A. Di Bartolomeo, "The role of contact resistance in graphene field-effect devices," arXiv., pp. 1-70, 2017.

[26] S. Naghdi, G. Sanchez-Arriaga, and K.Y. Rhee, "Tuning the work function of graphene toward application as anode and cathode," J Alloys Compd., vol. 805, pp. 1117-34, 2019.

[27] G. Giovannetti, P.A. Khomyakov, G. Brocks, V.M. Karpan, J.V.D Brink, and P.J. Kelly, "Doping Graphene with Metal Contacts," vol. 026803, pp. 4-7, 2008.

[28] CHARGE solver introduction, "Lumerical Support," [online]. Retrieved from https://support.lumerical.com/hc/en-us/articles/360034917693CHARGE-solver-introduction\%0A

[29] M. Liu, X. Yin, E. Ulin-Avila, B. Geng, T. Zentgraf, and L. Ju, "A graphene-based broadband optical modulator," Nature, 2011.

[30] L. Zhao, R. He, K.T. Rim, T. Schiros, K.S. Kim, and H. Zhou, "Visualizing individual nitrogen dopants in monolayer graphene," Science, vol. 333, no. 6045, pp. 999-1003, 2011.

[31] M. Pykal, P. Jurečka, F. Karlický, and M. Otyepka, "Modelling of graphene functionalization," Physical Chemistry Chemical Physics, 2016.

[32] S.J. Han, Z. Chen, A.A. Bol, and Y. Sun, "Channel-length-dependent transport behaviors of graphene field-effect transistors," IEEE Electron Device Lett., 2011. 